\title{
Antibiotic Prophylaxis In Preventing Surgical Site Infection In Patients Undergoing Lichtenstein's Hernioplasty
}

Rizwanullah Junaid Bhanbhro, Khalil Ahmed Almani, Sadia Kazi

ABSTRAC̄T

Objective: To determine the antibiotic prophylaxis in preventing surgical site infection in patients undergoing Lichtenstein's hernioplasty.

Study design: Observational study

Place and Duration: Department of surgery, ISRA University Hospital, Hyderabad. from December 2015 to March 2017.

Materials and Methods: 120 cases of inguinal hernia planned for Lichtenstein's hernioplasty were selected according to exclusion criteria and divided into control and antibiotic group. Pre- operative patient history, physical examination and inguinal hernia examination was performed. Prophylactic antibiotic ceftriaxone $(1000 \mathrm{mg})$ was given over night of surgical procedure. Statistical software SPSS 21.0 was used for data analysis at 95\% $\mathrm{CI}(P<0.05)$.

Results: Mean \pm SD age was noted as $39.51 \pm 7.56$ years ( $15-61$ years). Of 120 , who underwent Lichtenstein's hernioplasty the direct and indirect hernias were noted in $35(29.16 \%)$ and $33(27.5 \%) \& 25(20.83 \%)$ and $27(22.5 \%)$ of control and antibiotic groups respectively. Of 120 subjects, the SSI was noted in $17(14.1 \%)$ in control and $5(4.16 \%)$ in antibiotic group. Cumulative SSI in 120 cases was 22 (18.33\%).

Conclusion: The present study reports low incidence of surgical site infection with antibiotic prophylaxis in Lichtenstein's mesh repair in open inguinal hernioplasty.

Key words: Antibiotic Prophylaxis, Surgical site infection, Lichtenstein's mesh repair, Inguinal Hernia

\section{INTRODUCTION:}

Inguinal hernia is a common surgical problem. Its incidences rise with age. Male predisposition is common. An incidence of 368/100,000 for male and 44/100,000 for female has been reported ${ }^{1}$. The incidence changes to 194 to $648 / 100,000$ for male and 28 to 108/100,000 for female in older age group ${ }^{1,2}$. Weakness of anterior abdominal wall muscles and raised intra-abdominal pressure are contributing casues. Constipation, chronic cough and benign prostate hypertrophy raise the abdominal pressure. Inguinal hernia causes swelling in inguinal area and distress ${ }^{2}$. Sole therapeutic option for inguinal hernia is a surgical repair. This prevents the hernia complication of bowel strangulation and intestinal obstruction $^{3}$. Surgical repair of inguinal hernia repair is one of most common surgical procedure performed throughout the World ${ }^{4}$. Clinical trials have conceded the Lichtenstein's hernioplasty as the "gold standard" surgical procedure for

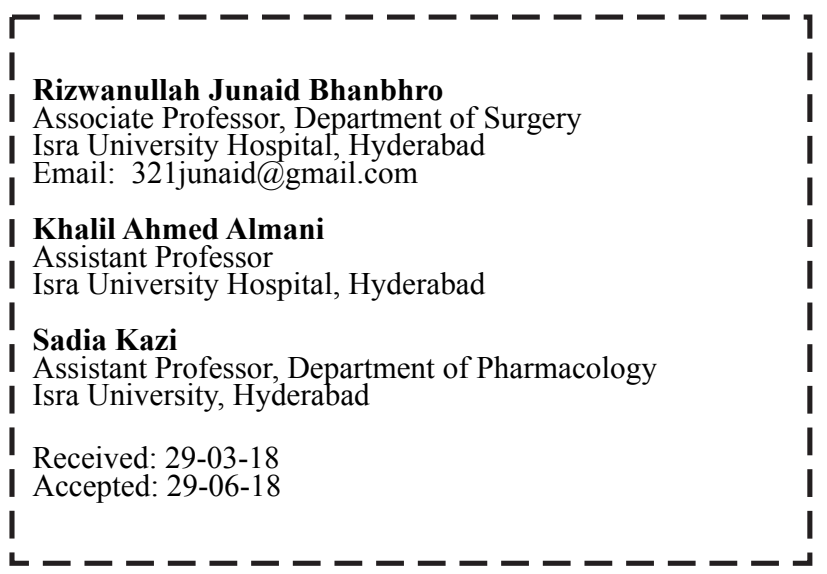

the inguinal hernia $a^{5-7}$. Inguinal hernia repair is considered to be a clean surgical procedure so antibiotic prophylaxis is not indicated. Contrary to this the surgical site infection (SSI) is most common complication of inguinal hernia repair. This is because of mesh which is used in hernia repair that functions as foreign body, hence needs prophylactic antibiotics pre- peratively ${ }^{8}$. Role of antibiotic prophylaxis in Lichtenstein's mesh repair is a controversial area. A Cochrane meta- analysis concluded the antibiotic prophylaxis is neither recommended nor discarded in Lichtenstein's mesh repair. In developing countries the SSI is a common surgical problem which cost a lot despite poor economy. Irrational use of antibiotics in Lichtenstein's mesh repair increases the financial burden. This is of particular concern in developing countries like Pakistan where hospitals have limited funds, patients are non- affording and are treated free of cost. On the contrary, post procedure SSI creates more financial burden on the patients ${ }^{\mathbf{8}, 9}$. As the current knowledge has created confusing results with conflicting views, hence the topic needs further research. The present prospective study was planned to define the role of prophylactic antibiotic in Lichtenstein's mesh repair in open inguinal hernioplasty at our tertiary care hospital.

\section{PATIENTS AND METHODS:}

Ethical approval for the present prospective observational study was taken from the institute. The study was conducted at the surgical wards of Isra University Hospital. It covered duration from December 2015 to March 2017. A sample of 120 inguinal hernias was selected according to the inclusion and exclusion criteria. Purposive sampling was used to 
collect sample. Patients with unilateral inguinal hernia planned for Lichtenstein's hernioplasty were selected. Age $>15$ years and $<60$ years, healthy male with unilateral inguinal hernia and planned Lichtenstein's hernioplasty were included in the study protocol. Patients with recurrent inguinal hernia, bilateral inguinal hernia, antibiotic intake, female gender, and those with pulmonary tuberculosis and chronic liver disease were excluded. 120 subjects were divided into 2 groups; control (not received prophylactic antibiotic) and antibiotic group (received prophylactic antibiotic). Prophylaxis antibiotic ceftriaxone (1000 mg) was given over night of surgical procedure. Control group was administered normal saline as placebo. Surgical site was examined on $2^{\text {nd }}$ post operative day. Follow up was followed on $10^{\text {th }}, 20^{\text {th }}$ and $30^{\text {th }}$ day. Surgical site infection was noted in both groups. Pre- operatively, a written consent was signed by volunteers and they were informed that the antibiotic will be provided by the hospital, and there will be no extra burden on their pockets. A pre- structured proforma was designed for collection of data from volunteers. Statistical software SPSS 21.0 was used for data analysis (IBM, incorporation, USA). Gaussian distribution of continuous variables was checked by "Kolmorgov- Smirnov test". Continuous and categorical variables were analyzed by "Student t- test" and "Chi square test" respectively. All statistical analysis was calculated at 95\% CI $(P<0.05)$ for significance.

\section{RESULTS:}

Mean \pm SD age of study population was noted as $39.51 \pm$ 7.56 years $(15-61$ years). Majority of subjects were found in $3^{\text {rd }}, 4^{\text {th }}$ and $5^{\text {th }}$ decades of life $(\mathrm{P}>0.05)$. In controls and antibiotic groups $17(14.1 \%)$ and $15(12.5 \%)$ were noted in $3^{\text {rd }}$ decades, $19(15.83 \%)$ and $20(16.66 \%)$ in $4^{\text {th }}$ decade, and $11(9.16 \%)$ and $10(8.33 \%)$ were noted in $5^{\text {th }}$ decade respectively. Table 2 shows the frequency of types of inguinal hernia in the control and antibiotic groups. Of 120, who underwent Lichtenstein's hernioplasty the direct and indirect hernias were noted in $35(29.16 \%)$ and $33(27.5 \%) \& 25$ $(20.83 \%)$ and $27(22.5 \%)$ of control and antibiotic groups respectively. Majority of subjects, both control and antibiotic group show right inguinal hernia. Of 120 subjects, the SSI was noted as $17(14.1 \%)$ in control and $5(4.16 \%)$ in antibiotic group. Cumulative SSI in total study population was 22 $(18.33 \%)$ as shown in table 3 .

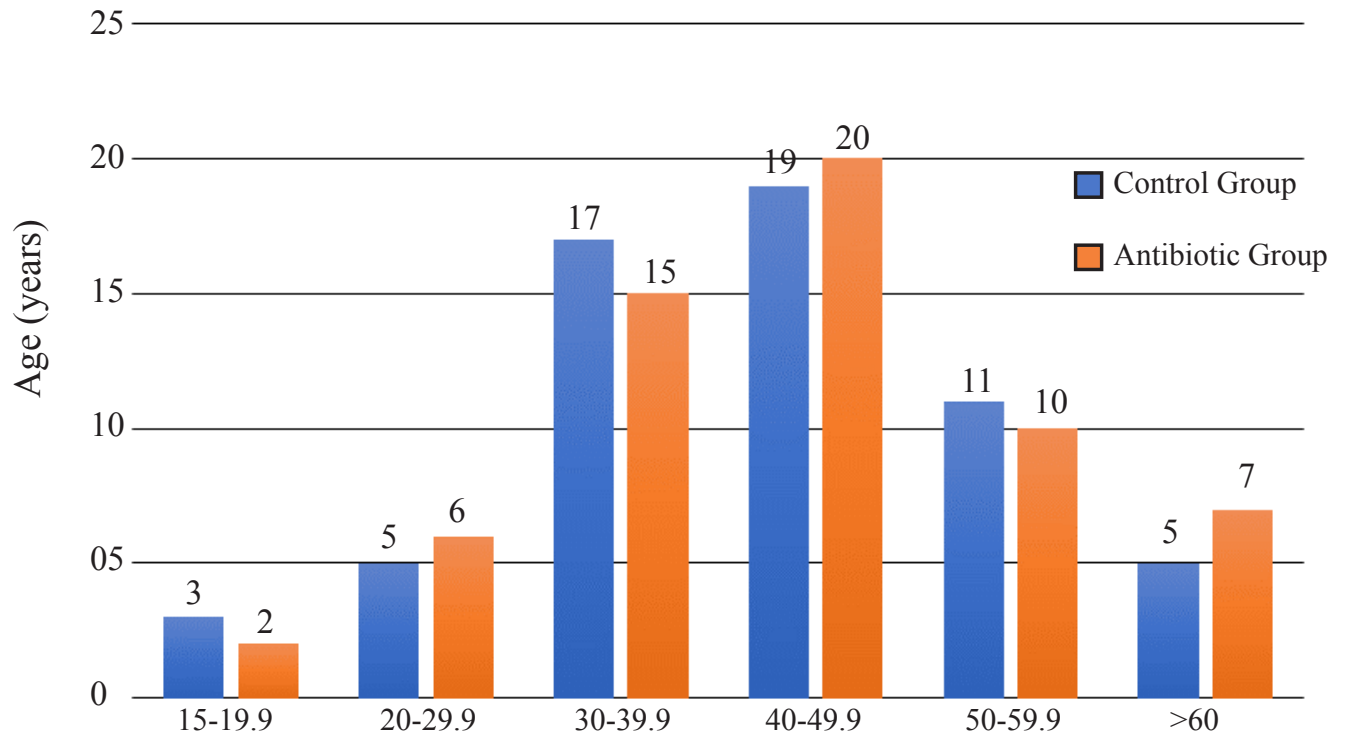

Fig 1. Age distribution of study subjects

\begin{tabular}{|c|c|c|c|}
\hline Types & Control Group & Antibiotic Group & P-value \\
\hline Direct & $35(29.16 \%)$ & $33(27.5 \%)$ & \multirow{2}{*}{0.093} \\
\cline { 1 - 3 } Indirect & $25(20.83 \%)$ & $27(22.5 \%)$ & \\
\hline Total & $60(50 \%)$ & $60(50 \%)$ & \\
\hline
\end{tabular}

Table. 2. Types of Inguinal Hernia in study subjects $(n=120)$

\begin{tabular}{|c|c|c|c|}
\hline SSI & Control Group & Antibiotic Group & P-value \\
\hline Yes & $17(14.1 \%)$ & $5(4.16 \%)$ & \multirow{2}{*}{0.093} \\
\hline No & $43(71.66 \%)$ & $55(45.83 \%)$ & \multirow{2}{*}{0} \\
\hline Total & $60(50 \%)$ & $60(50 \%)$ & \\
\hline
\end{tabular}

Table. 3. Surgical site infection (SSI) in study subjects $(\mathrm{n}=120)$ 


\section{DISCUSSION:}

The present prospective study reports on the SSI in Lichtenstein's mesh repair in open inguinal hernioplasty. Mean $\pm \mathrm{SD}$ age in total study population was noted as 39.51 \pm 7.56 years $(15-61$ years $)$. Majority of subjects were found in $3^{\text {rd }}, 4^{\text {th }}$ and $5^{\text {th }}$ decades of life $(\mathrm{P}>0.05)$. These findings are supported by previous studies. ${ }^{10,11}$ In controls and antibiotic groups $17(14.1 \%)$ and $15(12.5 \%)$ were noted in $3^{\text {rd }}$ decades, $19(15.83 \%)$ and $20(16.66 \%)$ in $4^{\text {th }}$ decade, and $11(9.16 \%)$ and $10(8.33 \%)$ were noted in $5^{\text {th }}$ decade respectively. These findings are in keeping with previous reports $^{12,13}$. Of 120 , who underwent Lichtenstein's hernioplasty the direct and indirect hernias were noted in $35(29.16 \%)$ and $33(27.5 \%) \& 25(20.83 \%)$ and $27(22.5 \%)$ of control and antibiotic groups respectively. These findings are supported by previous studies ${ }^{9,12,13}$. In present study, SSI was $17(14.1 \%)$ in control and $5(4.16 \%)$ in antibiotic group. Cumulative SSI in total study population was $22(18.33 \%)$ as shown in table 3 . The findings are in agreement with previous study ${ }^{9}$ that reported $12 \%$ incidence of SSI. Our incidence of $18.33 \%$ is slightly higher. While other previous studies have reported incidence of SSI of $8.33 \%$ and $8.7 \%$ respectively ${ }^{14,15}$ which is in disagreement with the present study. It has been said there is no reliable data regarding the SSI infection rates in hospitals in the developing countries. The present study is a contribution of SSI in inguinal hernioplasty which enlightens the reality in developing countries. A previous study reported SSI incidence following mesh repair of inguinal hernia ranges from $0 \%$ to $9 \%{ }^{16}$. This much difference of SSI incidence is probably due to the health provision facilities which are different in developing and developed countries. Other factors could be contributing such as the study design, different geographical areas, different study populations, physical status, nutritional status, operation theaters sterilizations, duration of follow-up and surgical procedure (mesh repair versus non-mesh repair) ${ }^{\mathbf{1 7}}$. In present study, the association of incidence of SSI was not analyzed with other risk factors such as the hospital stay, age, operation theater environment, instrumental autoclaving facilities, etc. In the present study, incidence of SSI in Lichtenstein's mesh repair in open inguinal hernioplasty was low in antibiotic group $5(4.16 \%)$ compared to 17 (14.1\%) in control group. The total incidence of $18 \%$ of SSI is slightly higher that previous studies ${ }^{19,20}$. This could be due to different study populations, surgical facilities and small sample size. The findings of present study are in disagreement with previous study ${ }^{19}$ that reported SSI incidence of $1.8 \%$ in the control group and $1.6 \%$ in those received prophylactic antibiotics. This previous study ${ }^{\mathbf{1 9}}$ concluded the prophylaxis antibiotic do not protect against SSI that is in contrast to the observations of present study. The findings are also in disagreement with previous studies ${ }^{\mathbf{2 0 , 2 1}}$ who reported SSI incidence of $3.3 \%$ and $1.7 \%$ in the control and antibiotic group respectively. The reason is clear that these studies have been reported from developed countries where health facilities are available at the climax. The present study is a contribution to the surgical site infection in Lichtenstein's mesh repair in open inguinal hernioplasty and enlightens the reality in developing countries. The present study suggests the prophylactic antibiotic therapy decreases the chances of surgical site infection.

\section{CONCLUSION:}

Surgical site infection incidence was high in present study. The present study reports low incidence of surgical site infection in Lichtenstein's mesh repair in open inguinal hernioplasty with antibiotic prophylaxis from the evidence based findings of present study the routine use of prophylactic antibiotic decrease the incidence of SSI in mesh hernia repair.

\section{REFERENCES:}

1. Ramirez T, Jones T, Kuchena A, Ali SM. Incidence of inguinal hernia repairs in Olmsted County, $\mathrm{MN}$ : a population-based study. Ann Surg 2013; 257(3): 520-6.

2. Amato B, Compagna R, Fappiano F, Rossi R, Bianco T, Danzi $M$, et al. Day-surgery inguinal hernia repair in the elderly: single centre experience. BMC Surgery 2013; 13(2):28.

3. Amato B, Compagna R, Della Corte GA, Martino G, Coretti G, Rossi R, et al. Feasibility of inguinal hernioplasty under local anaesthesia in elderly patients. BMC Surg 2012; 12(1):2.

4. Deysine M. Post mesh herniorrhaphy wound infections: can they be eliminated? Int Surg 2005; 90 (Suppl. 3):S40-4.

5. Rutkow IM. Demographic and socioeconomic aspects of hernia repair in the United States in 2003. Surg Clin North Am 2003; 83:1045.

6. Vrijland WW, van den Tol MP, Luijendijk RW. Randomized clinical trial of non-mesh versus mesh repair of primary inguinal hernia. Br J Surg 2002; 89:293-7.

7. Nordin P, Bartelmess P, Jansson C. Randomized trial of Lichten shouldice hernia repair in general surgical practice. Br J Surg 2002; 89:45-9.

8. Bendavid R. Complications of groin hernia surgery. Surg Clin North Am 1998; 78:1089-103.

9. Alagarsamy GS, Ramasamy R. The efficacy of antibiotic prophylaxis in preventing SSI (surgical site infection) in patients undergoing Lichtenstein's hernioplasty at our tertiary care centre. Int Surg J 2017; 4:1922-5.

10. Zaheer A, Sujeet KB, Monika K, Rakesh B. desarda no mesh repair versus Lichtenstein open mesh repair of inguinal hernia: a comparative study. Journal of Evolution of Medical and Dental Sciences 2015; 4(77):13279-82.

11. Afzal A, Ali R, Yousaf S. Outcomes of Desarda Vs Lichtenstein Repair for Inguinal Hernia in Terms of Operative Time, Seroma Formation, Return to Normal Activity and Cost. Pak J Med Health Sci 2017;11(1):93-6.

12. Al-Fatah M A, Al-Sheemy G, Al-Aal A. Desarda inguinal herniorraphy. J Asian Stud 2016;12(1):132-140.

13. Shehzad B, mohammad Omar A, Yaseen Rafi. desarda technique for inguinal hernia repair, a multicenter experience. Pak J Med Health Sci 2015;9(1):311-313.

14. Vinoth N, Karthikeyan CRM, Parmar H. Open inguinal 
hernioplasty: a prospective randomized clinical trial. Int'1 Arch Integ Med 2015;2(3)57-67.

15. Shankar VG, Srinivasan K, Sistla SC, Jagdish S. Prophylactic antibiotics in open mesh repair of inguinal hernia: a randomized controlled trial. Int J Surg 2010;8:444-7.

16. Law DJ, Mishriki SF, Jeffery PJ. The importance of surveillance after discharge from hospital in the diagnosis of postoperative wound infection. Ann R Coll Surg Engl 1990;72(3):207-9.

17. Terzi C. Antimicrobial prophylaxis in clean surgery with special focus on inguinal hernia repair with mesh. J Hosp Infect 2006;62(4):427-36.

18. Yerdel MA, Akin EB, Dolalan S. Effect of single-dose prophylactic ampicillin and sulbactam on wound infection after tension-free inguinal hernia repair with polypropylene mesh. Ann Sur 2001;233:26-33.
19. Aufenacker TJ, van Geldere D, vanMesdag T. The Role of Antibiotic Prophylaxis in Prevention of wound infection after lichtenstein open mesh repair of primary inguinal hernia multicenter double controlled trial. Ann Surg 2004;240:95561.

20. Perez AR, Roxas MF, Hilvano SS. A randomized, doubleblind, placebo controlled trial to determine effectiveness of antibiotic prophylaxis for tension free mesh herniorrhaphy. J Am Coll Surg 2005;200:393-9.

21. Tzovaras G, Delikoukos S, Christodoulides G, Spyridakis M, Mantzos F, Tepetes K, et al. The role of antibiotic prophylaxis in elective tension-free mesh inguinal hernia repair: results of a single-centre prospective randomised trial. Int J Clin Pract 2007;61(2):236-9. 